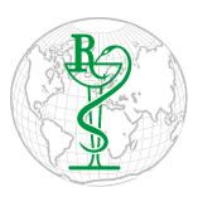

INDO GLOBAL JOURNAL OF

PHARMACEUTICAL SCIENCES

ISSN 2249- 1023

\title{
Recent Advancements in the Treatment of Diabetic Retinopathy
}

\author{
Hemant Bhola ${ }^{1}$, Amar Ranjan ${ }^{2}$, Smriti Khatri ${ }^{3}$ \\ ${ }^{1}$ Spectrum Institute of Pharmaceutical Sciences and Research, Greater Noida, UP, India \\ ${ }^{2}$ Cancer Institute, All India Institute of Medical Sciences (AIIMS), New Delhi-1 10029, India
}

${ }^{3}$ Turacoz Healthcare Solutions, Gurgaon, India

Address for Correspondence: Smriti Khatri, smritidua3@gmail.com

\begin{abstract}
Received:
15.06.2019

Accepted:

02.01.2020

Published:

15.12.2020

\section{Keywords}

Diabetic

Retinopathy;

Vascular

endothelial

growth factor

(VEGF);

Diabetic macular

edema (DME).
\end{abstract}

\begin{abstract}
Diabetic Retinopathy (DR) remains the most widely recognized challenge of the micro vascular problems of diabetes, despite recent advancements in the treatment of DR worldwide. The drugs that block the actions of vascular endothelial growth factor (VEGF) and corticosteroids, have shifted the goal of DR treatment from stabilization of vision to improvement. Although aggressive treatment improves vision in most patients, but many still do not achieve reading and driving vision. For over 30 years laser was the mainstay for the treatment of diabetic macular edema (DME), but several important management questions and treatment deficiencies remain unanswered with the laser therapy. This review summarizes updates on diagnosis, imaging, systemic factors, and glycemic control, vitreolysis, surgery, limitations and current treatment options such as neurovascular unit, which includes ranibizumab, aflibercept, bevacizumab, dexamethasone, fluocinolone acetonide and important clinical treatments in the management of DR. (C) 2020 iGlobal Research and Publishing Foundation. All rights reserved.
\end{abstract}

Cite this article as: Bhola, H.; Ranjan, A.; Khatri, S. Recent advancements in the treatment of diabetic nephropathy. Indo Global J. Pharm. Sci., 2020; 10(4): 6-9. DOI: http://doi.org/10.35652/IGJPS.2020.10402 .

\section{INTRODUCTION}

The worldwide prevalence of diabetes mellitus is predicted to increase adequately in the coming years. The approximate increment is 382 million in 2013 to 592 million in 2035.[1,2] Diabetic patients suffer several life-restricting problems, which may include micro vascular-related retinopathy, nephropathy, macrovascular related retinopathy, and ischemic heart disease. Diabetic Retinopathy (DR) is the most widely recognized micro vascular problems of diabetes. [3] DR presently influences around 100 million people globally and is ready to turn into an ever-increasing health problem. DR related visual impairment and blindness raised by $64 \%$ \& $27 \%$ as seen in the year between 1990 and 2010. [4] In spite of earlier searches in the diagnosis and treatment of diabetic retinopathy, this problem remains a dedicated challenge to patients and physicians. Moreover, the majority of the population globally does not have access to specialized care and affordable drugs. A dreadful challenge to the healthcare system is represented by the treatment, prevention, and diagnosis of DR. This review aims to provide diagnosis, imaging, limitations, and important clinical updates in the management of DR.

\section{AN UPDATE ON DIAGNOSIS AND IMAGING}

The root cause of DR is complex and the primary contributor is intense capillary non- perfusion and retinal ischemia. The communication molecules insulin-like growth factor-1, platelet- derived growth factor, angiopoietin and Vascular Endothelial Growth Factor (VEGF) plays a vital role in the consequent advancement of microangiopathy. [5] Evidence from the recent clinical data recommends that neurodegenration is an early event within the pathological process of DR.6,7 Imaging modalities particularly Optical Coherence Tomography (OCT) and Fluorescein Angiography (FA), currently plays an important role in the identification and management of complications of DR, especially for Diabetic Macular Edema (DME) and subtle NV. Modern procedure of OCT, together with Optical Coherence Tomography Angiography (OCTA) expands by utilizing variation in phases and speed of the light signal from vascular structure. [6-8] OCTA can resolve vascular subtleties not attainable by the ordinary FA, for instance, the superficial capillary plexus. [9] 


\section{Indo Global Journal of Pharmaceutical Sciences, 2020; 10(4): 6-9}

\section{Systemic factors and Glycemic control}

The United Kingdom prospective diabetes study and the Diabetes Control and Complications Trial (DCCT) validates that glycemic control decreases micro vascular problems for type 1 and type 2 diabetes. [10] An ongoing 30 years follow up of DCCT and the epidemiology of diabetes interventions and complication study reveals the importance of HaemoglobinA1c (HbA1c) control, coming out with a 50\% risk reduction of retinopathy progression in intensive glycemic control patients, in spite a consequent increase (and alternative decrease in standard control patients) to a mean $\mathrm{HbA1c}$ value of $8 \%$. [11,12]

\section{Vitreolysis and Surgery}

The vitreoretinal medical procedure is the standard treatment of many visual inconveniences of DR. Macular Edema from vitreous traction or epiretinal membranes can be experienced by diabetic patients and both of these can be treated surgically. [13]

\section{Laser}

The study of diabetic retinopathy gives an explanation of the reduction in some vision loss in patients with high risk Proliferative Diabetic Retinopathy (PDR) followed by immediate treatment with Pre Retinal Photocoagulation (PRP). [14] The Early Treatment Diabetic Retinopathy Study (ETDRS) indicates a 50\% reduction in vision loss in patients having Clinically Significant Diabetic Macular Edema (CSDME) who have undergone instant focal laser photocoagulation . [15]

\section{Limitations and Current Treatment Options}

Laser photocoagulation intravitreal injections of anti-VEGF and steroidal agents are the intra ocular surgery procedure for diabetic eye illness. Modern therapeutic paradigms targets for treatment of advanced diseases, by developing PDR or DME. In the current scenario phase 3 clinical trials have indicated the prevalence of intravitreous anti-VEGF injections to laser monotherapy in lowering vision loss and bettering the rates of vision gaining eye with DME. [16-18] Aflibercept, Bevacizumab, and Ranibizumab are frequently been utilized as anti-VEGF agents which have shown an efficiency in correcting vision by 1 and 2 years of treatment of DME [1920] Anti-VEGF medical care is very efficient in relapsing retinal neovascularization in eyes with PDR. [21]

\section{Neurovascular Unit}

Enhanced retinal imaging with early cell changes in the diabetic retina has prompted a conceptualization that DR can be seen as a disorder of the retinal neurovascular unit. It alludes to the practical coupling and interdependency of neurons, glia and vascular which integrate to direct ordinary retinal function. [22]

\section{Ranibizumab}

The Anti-VEGF agents have revolutionized the management of DR. Under the trials of ranibizumab for diabetic Macular Edema (Ride and Rise) two doses $0.5 \mathrm{mg}$ and $0.3 \mathrm{mg}$ has been investigated for the monthly use for the treatment of DME. [23] First-line agents to treat central-involving DME are intravitreal VEGF inhibitors.

\section{Aflibercept}

One of the world's most widely used agent Aflibercept is a fusion of the Human Ig GFc region and the extracellular VEGF receptor ligand-binding region combines to VEGF-A, VEGF-B, Placental growth factor-I (PLGF-1) \& (PLGF-2). [24] FDA approves it for the treatment of DME \& DR.

\section{Bevacizumab}

Bevacizumab (Avastin, Genetch) comprise of a full-length humanized murine monoclonal antibody that binds to VEGFA. [25] FDA has not approved it for the treatment of DME and DR. the efficacy of bevacizumab versus focal laser for DME has been examined by the intravitreal bevacizumab or laser therapy in the management of DME.

\section{Dexamethasone \& Fluocinolone acetonide}

In spite of the predominance of anti-VEGF agents, there is yet a role to carry out for intravitreal corticosteroids. Steroids firstly inhibit leukostasis and then improve the barrier function of tight junctions and in last modify the release of local inflammatory factors, which may include VEGF. [26]

\section{CONCLUSION}

Corticosteroids are initials to take an area as second-line medical aid for patients insensitive to anti-VEGF agent and focal optical device. This is mainly relevant for pseudophakic patients who exceed a steroid IOP challenge. The continued outcome of these current studies on pharmacological agents is that early diagnosis of DME in NPDR or PDR, as is crucial to stop and sometimes converse retinopathy. Despite significant growth has been made in the diagnosis of DR and its development, the initial strategy DR should be ardent. AntiVEGF specialists have treated into primary line operators for the diagnosis of DME. It has been proved that intravitreal VEGF inhibitors are effective in DR regression. VEGF inhibitors give the effective result for the treatment cure vision in individuals with DR. Future planning should target on alternative therapeutic agents or an invasive delivery method. During the rising stages of DR, an important role is played by neurodegeneration, also intravitreal anti-VEGF injections have 
Indo Global Journal of Pharmaceutical Sciences, 2020; 10(4): 6-9

shown an aggressive approach. At this point of time topical therapies, for instance endogenous neuroprotective substances holds an exciting promise. [27]. We have witnessed dramatic improvements in the treatment of DR with the introduction of potent pharmacotherapy over the past decade. As we better understand the capabilities of available drugs and integrate them with treatments such as laser and surgery, and add new pharmacologic drugs to our treatment paradigms when they receive FDA approval, the future treatment for DR appears increasingly promising.

\section{FUTURE DIRECTIONS}

Concept of protective mechanism: Although a significant research effort has been conducted/ supervised to recognize the pathogenic pathways, adding to the beginning and progression of DR, a developing paradigm is the significance if endogenous systems that secure against DR. $[28,29]$ This concept is actively being supported by the Joslin medalist study of 50 year, in which about 1000 individuals had taken part with Type 1 Diabetes with a time period of 50 years or more and approximately $40 \%$ of these patients were treated with diabetes before glycemic control, still they had no or mild DR. [30] Many protective factors opted in the DR, which may include Pigment Epithelium Derived Factor (PEDF),Somatostatin, NFE2-related factor 2 (Nrf2) have been identified. [31-33]

New therapeutic angles in diabetic retinopathy: While the past decade has shown vital enhancements within the treatment choices for DR, extra therapies are required urgently. Current treatments are coordinated solely towards progressive phases of DR, usually when invariable harm has resulted, in this manner medication that are protective or address early pathology are desirable. Anti-VEGF therapy is just half way compelling against DME and the recognizable proof of additional, VEGF free pathogenic particles, in this condition could prompt new medications that have better protected vision. [34] TNF- $\alpha$ and lipoprotein- associated phospholipase A2(LP-PLAA2) are the additional molecular targets, identified for pharmacological inhibitors. [35-36]

Precision Medicine: Patients with diabetes inhibit long variations during their treatment of retinopathy. It includes the pace of progressions as well as clinical manifestations. For example, some patients show a high tendency to treat DME while few may move towards PDR. Some individuals give better result for anti-VEGF therapy, while some shows moderate or poor response, this may lead to the variable result of the treatment. [37] A robust development pipeline of new DME drugs in phase I and II testing is available. Their potential uses vary from disease modulation in patients with early DR, to monotherapy or combination therapy for patients and can be a better treatment option for patients with DR in near future.

\section{ACKNOWLEDGEMENT}

Not declared.

\section{DATA AVAILABILITY}

Not declared.

\section{CONFLICTS OF INTEREST}

The authors declare no conflict of interest in this research article.

\section{FUNDING SOURCE}

No external funding declared.

\section{REFERENCES}

1. Guariguata L, Whiting DR, Hambleton I, Beagley J, Linnenkamp U, Shaw JE. Global estimates of diabetes prevalence for 2013 and projections for 2035. Diabetes Res Clin Pract. 2014;103(2):137-149.

2. Nanditha A, et al. Diabetes in Asia and the Pacific: Implications for the Global Epidemic. Diabetes Care. 2016;39(3):472-485.

3. Antonetti DA, Klein R, Gardner TW. Diabetic retinopathy. N Engl J Med. 2012;366(13):1227-1239.

4. Leasher JL, et al. Global Estimates on the Number of People Blind or Visually Impaired by Diabetic Retinopathy: A Metaanalysis From 1990 to 2010. Diabetes Care. 2016;39(9):16431649.

5. Simó R, Hernández C: Novel approaches for treating diabetic retinopathy based on recent pathogenic evidence. Prog Retin Eye Res. 2015; 48: 160-80.

6. Stem MS, Gardner TW: Neurodegeneration in the pathogenesis of diabetic retinopathy: molecular mechanisms and therapeutic implications. Curr Med Chem. 2013; 20(26): 3241-50.

7. Simó R, Hernández C, European Consortium for the Early Treatment of Diabetic Retinopathy (EUROCONDOR): Neurodegeneration in the diabetic eye: new insights and therapeutic perspectives. Trends Endocrinol Metab. 2014; 25(1): 23-33.

8. An L, Wang RK: Volumetric imaging of microcirculations in human retina and choroids in vivo by optical microangiography. In: Tuchin VV, Wang LV, editors. San Jose, CA: SPIE; 2008; 68550A.

9. Spaide RF, Klancnik JM Jr, Cooney MJ: Retinal vascular layers imaged by fluorescein angiography and optical coherence tomography angiography. JAMA Ophthalmol. 2015; 133(1): 45-50.

10. UK Prospective Diabetes Study (UKPDS) Group. Intensive blood-glucose control with sulphonylureas or insulin compared with conventional treatment and risk of complications in patients with type 2 diabetes (UKPDS 33). The lancet. 1998 Sep 12;352(9131):837-53.

11. Holman RR, Paul SK, Bethel MA, et al.: 10-year follow-up of intensive glucose control in type 2 diabetes. $\mathrm{N}$ Engl $\mathrm{J}$ Med. 2008; 359(15): 1577-89.

12. Nathan DM, Bayless M, Cleary P, et al.: Diabetes control and complications trial/epidemiology of diabetes interventions and complications study at 30 years: advances and contributions. Diabetes. 2013; 62(12): 3976-86.

13. Aaberg TM: Pars plana vitrectomy for diabetic traction retinal detachment. Ophthalmology. 1981; 88(7): 639-42. 


\section{Indo Global Journal of Pharmaceutical Sciences, 2020; 10(4): 6-9}

14. Diabetic Retinopathy Study Research Group. Preliminary report on effects of photocoagulation therapy. American journal of ophthalmology. 1976 Apr 1;81(4):383-96.

15. Photocoagulation for diabetic macular edema. Early Treatment Diabetic Retinopathy Study report number 1. Early Treatment Diabetic Retinopathy Study research group. Arch Ophthalmol. 1985; 103(12): 1796-806.

16. Diabetic Retinopathy Clinical Research Network, et al. Randomized trial evaluating ranibizumab plus prompt or deferred laser or triamcinolone plus prompt laser for diabetic macular edema. Ophthalmology. 2010;117(6):1064-1077.e35.

17. Brown DM, et al. Long-term outcomes of ranibizumab therapy for diabetic macular edema: the 36-month results from two phase III trials: RISE and RIDE. Ophthalmology. 2013;120(10):2013-2022.

18. Heier JS, et al. Intravitreal Aflibercept for Diabetic Macular Edema: 148-Week Results from the VISTA and VIVID Studies. Ophthalmology. 2016;123(11):2376-2385.

19. Wells JA, Glassman AR, Ayala AR, Jampol LM, et al. Aflibercept, bevacizumab, or ranibizumab for diabetic macular edema. The New England journal of medicine. 2015 Mar;372(13):1193-203.

20. Wells JA, et al. Aflibercept, Bevacizumab, or Ranibizumab for Diabetic Macular Edema: Two-Year Results from a Comparative Effectiveness Randomized Clinical Trial. Ophthalmology. 2016;123(6):1351-1359.

21. Avery RL, et al. Intravitreal bevacizumab (Avastin) in the treatment of proliferative diabetic retinopathy. Ophthalmology. 2006;113(10):1695.e1-1695.15.

22. Gardner TW, Davila JR. The neurovascular unit and the pathophysiologic basis of diabetic retinopathy. Graefes Arch Clin Exp Ophthalmol. 2017;255(1):1-6.

23. Brown DM, Nguyen QD, Marcus DM, et al.: Long-term outcomes of ranibizumab therapy for diabetic macular edema: the 36-month results from two phase III trials: RISE and RIDE. Ophthalmology. 2013; 120(10): 2013-22.

24. Cai S, Bressler NM: Aflibercept, bevacizumab or ranibizumab for diabetic macular oedema: recent clinically relevant findings from DRCR.net Protocol T. Curr Opin Ophthalmol. 2017; 28(6): 636-43.

25. Cai S, Bressler NM: Aflibercept, bevacizumab or ranibizumab for diabetic macular oedema: recent clinically relevant findings from DRCR.net Protocol T. Curr Opin Ophthalmol. 2017; 28(6): 636-43.

26. Boyer DS, Yoon YH, Belfort R Jr, et al.: Three-year, randomized, shamcontrolled trial of dexamethasone intravitreal implant in patients with diabetic macular edema. Ophthalmology. 2014; 121(10): 1904-14.

27. Hernández C, Dal Monte M, Simó R, et al.: Neuroprotection as a Therapeutic Target for Diabetic Retinopathy. J Diabetes Res. 2016; 2016: 9508541

28. Rask-Madsen C, King GL. Kidney complications: factors that protect the diabetic vasculature. Nat Med. 2010;16(1):40-41.

29. Rask-Madsen C, King GL. Vascular complications of diabetes: mechanisms of injury and protective factors. Cell Metab. 2013;17(1):20-33.

30. Sun JK, et al. Protection from retinopathy and other complications in patients with type 1 diabetes of extreme duration: the joslin 50-year medalist study. Diabetes Care. 2011;34(4):968-974.

31. Zhang SX, Wang JJ, Gao G, Shao C, Mott R, Ma JX. Pigment epithelium-derived factor (PEDF) is an endogenous antiinflammatory factor. FASEB J. 2006;20(2):323-325.

32. Simó $\mathrm{R}$, Hernández $\mathrm{C}$, European Consortium for the Early Treatment of Diabetic Retinopathy (EUROCONDOR). Neurodegeneration in the diabetic eye: new insights and therapeutic perspectives. Trends Endocrinol Metab. 2014;25(1):23-33.

33. Xu Z, et al. NRF2 plays a protective role in diabetic retinopathy in mice. Diabetologia. 2014;57(1):204-213.

34. Ford JA, Lois N, Royle P, Clar C, Shyangdan D, Waugh N. Current treatments in diabetic macular oedema: systematic review and meta-analysis. BMJ Open. 2013;3(3):e002269.

35. Sfikakis PP, et al. Infliximab for diabetic macular edema refractory to laser photocoagulation: a randomized, doubleblind, placebo-controlled, crossover, 32-week study. Diabetes Care. 2010;33(7):1523-1528.

36. Canning P, et al. Lipoprotein-associated phospholipase A2 (LpPLA2) as a therapeutic target to prevent retinal vasopermeability during diabetes. Proc Natl Acad Sci U S A. 2016;113(26):72137218.

37. Ashraf M, Souka A, Adelman R. Predicting outcomes to antivascular endothelial growth factor (VEGF) therapy in diabetic macular oedema: a review of the literature. Br J Ophthalmol. 2016;100(12):1596-1604.

Indo Global Journal of Pharmaceutical Sciences( ISSN 2249 1023; CODEN- IGJPAI; NLM ID: 101610675) indexed and abstracted in CrossRef (DOI Enabling), CNKI, UGC CARE Journal List, EMBASE (Elsevier), National Library of Medicine (NLM) Catalog (NCBI), ResearchGate, Publons (Clarivate Analytics), CAS (ACS), Index Copernicus, Google Scholar and many more. For further details, visit http://iglobaljournal.com 